\title{
El americanismo y la historia: Algunas pautas diacrónicas de creación de americanismos (léxicos) ${ }^{1}$.
}

\author{
Americanism and history : Some guidelines for creating diachronic \\ Americanisms ( lexical ).
}

José Luis Ramírez Luengo²

Ramírez L. José L. miradas Nº13 - 2015. ISSN: 0122 994X Págs 114 - 123

Recepción: Mayo 7 de 2015

Aprobación: Octubre 10 de 2015

Publicación: Diciembre 17 de 2015

\section{Resumen}

La definición del americanismo y el análisis concreto de estos elementos en momentos pasados cuentan ya con una bibliografía realmente notable, pero quizá no hayan sido tan estudiados, sin embargo, los procesos diacrónicos que terminan por dotar de este carácter a determinadas unidades léxicas a través de la historia.

Precisamente en esta línea, el presente trabajo investiga sobre ciertos aspectos teóricos que afectan al americanismo, su definición y sus principales características, para después poder exponer una metodología adecuada de análisis de estos elementos en su dimensión histórica y las pautas históricas que determinan su creación, así como, a partir de lo anterior, una taxonomía que establezca los diversos subtipos que se pueden distinguir cuando se atiende a su diacronía.

Palabras Clave: Historia de la lengua, español de América, léxico, americanismo, metodología.

1 Este trabajo es un resultado que guarda relación con las investigaciones desarrolladas por el Grupo de Investigación SEQUEHL (Seminario queretano de historia de la lengua), perteneciente a la Facultad de Lenguas y Letras de la Universidad Autónoma de Querétaro (Querétaro, México); concretamente, la creación de los americanismos.

2 Doctor en Filología Hispánica por la Universidad de Deusto (España) y especialista en historia del español, tanto en España como muy especialmente en América, actualmente se desempeña como Profesor a Tiempo Completo en la Facultad de Lenguas y Letras de la Universidad Autónoma de Querétaro (Querétaro, México). Correo electrónico: joseluis. ramirezluengo@gmail.com. 


\section{Abstract}

The definition of the concept americanism and the analysis of these lexical items (americanisms) in different moments of the History have been already considered several times in bibliography, but this may not be the situation of diachronic processes which provide this consideration to some words throughout History.

This paper aims to research about some theoretical aspects which concern the americanism, its definition and its main features, in order to explain not only an appropriate methodology for studying them, but also the historical patterns which produce their creation. Finally, a taxonomy that describes different subtypes of historical americanisms will be established.

Key Words: History of the Spanish Language, Latin American Spanish, lexicon, americanism, methodolody.

\section{Introducción}

Es probable que, en los estudios dedicados al léxico americano actual, el análisis de los americanismos constituya, si no la más atendida, al menos una de las cuestiones más revisadas por parte de los investigadores, interés que determina que la bibliografía que reflexiona acerca de cómo se puede definir este concepto o que se centra en el análisis concreto de estos elementos resulte realmente ingente, con trabajos de la importancia de Bohórquez (1984) para la primera de las cuestiones, o Buesa Oliver y Enguita Utrilla (1992), Moreno de Alba (1992) y Vaquero de Ramírez (1996) para la segunda. Por supuesto, esta importancia concedida en los estudios de dialectología sincrónica al vocabulario propiamente americano se defina como se defina lo propiamente americano- no es en absoluto llamativa, sino que responde a su declarada intención de describir los rasgos y fenómenos lingüísticos que caracterizan actualmente al español del Nuevo Mundo en contraste con otras variedades de la lengua, cuestión para la que el léxico diferencial tiene una relevancia más que notable.

Sorprende, por tanto, que en los estudios históricos no se haya concedido la misma importancia al estudio del americanismo que se descubre en la sincronía: si bien es verdad que existen numerosos trabajos donde se analiza el empleo de estos elementos en determinadas épocas pasadas -recuérdense, entre otros, a Hildebrandt (1961), Mejías (1980) o Frago (1999)-, también lo es que hasta el momento no se ha prestado atención a los procesos diacrónicos que terminan por dotar de este carácter a algunas unidades léxicas concretas, así como las implicaciones metodológicas que conlleva el estudio histórico de tales voces dialectalmente -en este caso, americanamente- marcadas.

Y tal abandono sorprende especialmente si se acepta que el objetivo fundamental de una historia del español de América ha de consistir, como se defiende en Ramírez Luengo (2012: 394), en ofrecer "una visión general que explique de qué manera -y en qué momento- se van configurando las variedades dialectales que existen hoy en tierras americanas, esto es, de qué manera y en qué momento se producen los procesos de dialectalización ", lo que en el caso del léxico conlleva la configuración de un mapa propio que va a identificar a una región "por medio de un conjunto de voces que, sean conocidas solamente en la zona o tengan un significado especial en ese lugar, constituyen un rasgo de identidad que distingue esa variedad del español de todas las demás del mundo hispánico" (Ramírez Luengo, 2012: 395)²; un mapa léxico, 
por tanto, que se conforma precisamente por todos esos vocablos diatópicamente marcados que, de forma general, se pueden agrupar bajo el epígrafe de americanismos.

\section{Marco de referencia}

No hace falta decir que el cumplimiento de los propósitos anteriores obliga en primer lugar a facilitar una definición clara y precisa del americanismo, a partir de la cual se pueda desarrollar la metodología de análisis diacrónico que se pretende: como es más que sabido, son múltiples los intentos $-\mathrm{a}$ veces incluso contradictorios (Ramírez Luengo, 2014: 2)- que han presentado diferentes estudiosos para definir este concepto $^{3}$, de manera que en esta ocasión, y teniendo en cuenta los objetivos que procura el análisis, se ha partido de la interpretación que Company (2007: 28-9) ofrece del mexicanismo, la cual, una vez adaptada al ámbito general americano, permite definir el americanismo lingüístico como 'el conjunto de voces, formas $o$ construcciones que son caracterizadoras del habla urbana, popular o culta, o ambas, de América y cuyo uso muy frecuente y cotidiano distancia la variedad americana respecto del español peninsular'; en consecuencia, se dará la consideración de americanismo a cada uno de los elementos aislados que conforman la unidad inmediatamente enunciada.

Más allá de la definición en sí, quizá lo más interesante del aporte de Company sean algunas de las consecuencias que derivan de ella, las cuales se pueden considerar principios metodológicos de primera importancia a la hora de estudiar de forma rigurosa el americanismo: en efecto, aspectos como la clara decantación por el uso -y no tanto el origen etimológico- a la hora de definir las voces que se enclavan dentro del concepto considerado o la existencia de diversos tipos de americanismos a resultas de las distintas formas como las unidades léxicas cumplen la definición anterior ${ }^{4}$ permiten no solo seleccionar de forma coherente los vocablos que se integran en la categoría aquí analizada, sino también clasificarlos de acuerdo con sus propias características ${ }^{5}$.

Ahora bien, la consecuencia más relevante de la definición de la profesora mexicana y de su decidida defensa del uso como criterio único para determinar qué vocablos se pueden considerar americanismos se descubre sin duda al aplicar la dimensión diacrónica al análisis de estos elementos: en efecto, la existencia más que reconocida de procesos de extensión léxica ${ }^{6}$ que provocan que determinadas voces cambien su área geográfica de empleo -bien expandiéndola, bien reduciéndola- en diferentes momentos históricos y el hecho reiteradamente mencionado de que solo el uso en una zona concreta convierte a un elemento en -ismo (americanismos, mexicanismo, etc.), conlleva de forma inevitable que la consideración de una voz como parte de esta categoría no se mantenga necesariamente inmutable a lo largo del tiempo, sino que pueda variar a través de la historia y dependa, por tanto, del factor cronológico; en otras palabras, la definición del americanismo a partir del uso impone el carácter dinámico de este concepto y la necesidad, por tanto, de apelar a lo diacrónico para poder valorar no tanto la inclusión - atemporal y estática-de un elemento léxico a esta categoría, sino más bien su pertenencia circunstancial a ella durante una época concreta de su devenir histórico ${ }^{7}$.

\section{Metodología}

En esta línea, el presente trabajo pretende meditar sobre ciertos aspectos teóricos que afectan al concepto ya mencionado, a su definición y a sus principales características, para a continuación poder exponer una 
metodología adecuada de análisis y una primera clasificación taxonómica de estos elementos en su devenir histórico.

Así pues, y partiendo de lo expuesto en los párrafos anteriores, se comenzará por realizar un acercamiento de tipo porcentual a ciertos elementos léxicos presentes en los corpus seleccionados que determine su carácter de americanismo en la sincronía actual; posteriormente se desarrollará un análisis semejante en el que se tendrá en cuenta la dimensión cronológica, con el propósito de describir el comportamiento de estos elementos a través del tiempo, así como los (posibles) procesos de extensión léxica que han experimentado en siglos pasados.

Por último, la comparación y el contraste de los casos considerados permitirá establecer una primera clasificación de los americanismos de acuerdo con las pautas de uso que muestran desde el punto de vista diacrónico, lo que resulta de interés no solo para los propósitos clasificatorios señalados más arriba, sino también para demostrar de forma fehaciente y objetiva el carácter dinámico de este concepto y la existencia de diferentes subtipos dentro de esta categoría cuyas peculiaridades será necesario estudiar con calma en próximos trabajos.

\section{Resultados}

A partir, pues, de la definición de Company (2007: 28-9) y de las implicaciones que presenta $^{8}$, es posible establecer una primera división básica entre los americanismos que, por su presencia y empleo exclusivo en el Nuevo Mundo a lo largo de su historia, siempre han poseído este carácter $\mathrm{y}$ aquellos que, por el contrario, han adquirido tan valoración en un momento concreto a causa de los procesos de extensión léxica mencionados más arriba; así, mientras que los primeros se pueden denominar americanismos no determinados diacrónicamente (AND), los segundos serán americanismos determinados diacrónicamente (ADD) y contarán con un punto de modificación valorativa (PMV) -entendido como el 'momento en el que determinado elemento adquiere un estatus diferente al que poseía previamente', en este caso el de americanismo- cuya detección será fundamental para entender de forma más profunda la configuración de la especificidad léxica americana.

A este respecto, los datos sincrónicos facilitados por CREA y muy especialmente los históricos que ofrecen CORDE y otros corpus lingüísticos de gran formato ${ }^{9}$ permiten exponer ante el lector una serie de unidades léxicas que se engloban en los grupos previamente establecidos: de esta manera, se encuadrarán dentro del primer tipo voces de origen amerindio como el quechuismo cuy 'conejillo de Indias más grande que el corriente (DAM, 2010: s.v. cuy) o el nahuatlismo zacate 'pasto, hierba usada como forraje' (DAM, 2010: s.v. zacate), de empleo sincrónica y diacrónicamente desconocido en España ${ }^{10}$, mientras que pertenecerán al segundo grupo otros vocablos que fueron de uso habitual en esta variedad del español y ya no lo son, tales como atorar 'obstruir', enojarse 'enfadarse' y todos aquellos que Moreno de Alba (2007: 190) denomina arcaísmos americanos o pseudoarcaísmos.

Dentro de estos últimos, quizá durazno 'melocotón' ejemplifique de manera clara los cambios que históricamente puede experimentar un término en cuanto a su estatus como americanismo y, por tanto, el carácter dinámico de la categoría que se viene postulando a lo largo de estas páginas: arcaísmo americano en palabras de Moreno de Alba (2007: 190), actualmente el empleo de este término 
resulta muy escaso y/o regional en Españacinco únicos ejemplos en CREA (Consulta: 09/04/2015)-, mientras que en América constituye una voz muy frecuente -239 casos en países como Argentina, Bolivia, Chile, Colombia, Ecuador, Paraguay, Perú, México y Venezuela, entre otros-, a resultas de lo cual el propio Diccionario de Americanismos lo reconoce como tal (DAM, 2010: s.v. durazno). Ahora bien, esta situación inmediatamente descrita no se ha mantenido inmutable a lo largo del tiempo, y los datos de CORDE (Consulta: 09/04/2015) permiten seguir perfectamente el proceso de transformación de este elemento, en una época perteneciente al español general, en vocablo característico de las variedades americanas de esta lengua.

\begin{tabular}{|l|l|l|l|}
\hline Siglo & España & América & \\
\hline XVI & $48(96 \%)$ & $2(4 \%)$ & \\
\hline XVII & $47\left(97^{\prime} 91 \%\right)$ & $1\left(2{ }^{\prime} 08 \%\right)$ & \\
\hline XVIII & $0(0 \%)$ & $18(100 \%)$ & \\
\hline XIX & $1\left(7^{\prime} 14 \%\right)$ & $13\left(92^{\prime}, 85 \%\right)$ & \\
\hline XX & $7\left(17^{\prime} 07 \%\right)$ & 3 & 4 \\
$\left(82^{\prime} 92^{\prime} \%\right)^{11}$ & \\
\hline CREA & & $5\left(2^{\prime} 04 \%\right)$ & $\begin{array}{l}2 \\
\left(97^{\prime} 95 \%\right)\end{array}$ \\
\hline
\end{tabular}

Tabla 1: Distribución del uso de durazno 'melocotón' según CORDE/CREA

Dejando de lado los porcentajes concretos $^{12}$, los datos de CORDE parecen justificar la idea de que durazno constituye, efectivamente, un americanismo en la sincronía actual, pero que ese carácter lo adquiere en un momento muy concreto, el siglo XVIII, que es la época en la que el uso de esta forma léxica -en principio generaldecae en España y, como resultado de su mantenimiento en América, se convierte en un término propio de estas variedades del español; salta a la vista, por tanto, que el término ahora analizado constituye un claro americanismo determinado diacrónicamente, y que tiene en la Centuria
Ilustrada su punto de modificación valorativa, de manera que solo a partir de este momento se puede considerar que pertenece a tal categoría.

\section{Discusión}

Por supuesto, no hace falta decir que el caso de durazno representa solo uno de los subtipos de americanismos determinados cronológicamente que existen, en concreto el que se denomina $A D D$ por pérdida léxica: como se indicó anteriormente, se trata de un elemento previamente común a ambas lados del Atlántico cuya desaparición en suelo europeo -sea total, como en este caso, o sea meramente parcial, como ocurre con enojarse ${ }^{13}$ trae por consecuencia su transformación en americanismo; junto a este tipo, en otras ocasiones los vocablos adquieren el estatus que aquí se analiza por alteración semántica, es decir, por la modificación del primitivo significado común a resultas de la adquisición de nuevos valores en América $-A D D$ por alteración semántica americana: tortilla 'alimento en forma de torta circular y aplanada, elaborado con masa de maíz o trigo (DAM, 2010: s.v. tortilla) - o como consecuencia de la desaparición de (alguno de) los valores iniciales en España $-A D D$ por alteración semántica española: prieto 'de tez morena oscura, de raza negra' (DAM, 2010: s.v. prieto)-, así como -desde el punto de vista teórico al menos- por la transformación divergente de los valores significativos en ambas variedades lingüísticas a la vez (ADD por alteración semántica doble).

En línea con lo anterior, y por lo que se refiere a la presencia más o menos habitual de estos subtipos en el español, es evidente la necesidad de llevar a cabo estudios que los documenten e inventaríen en su historia antes de llegar a conclusiones de ningún tipo, pero parece posible mantener 
-siquiera de forma intuitiva- que no todos ellos son igualmente frecuentes, de manera que, mientras que los $A D D$ por pérdida léxica y los $A D D$ por alteración semántica americana resultan notablemente abundantes, los ADD por alteración semántica española y alteración semántica doble parecen ser más escasos, muy especialmente estos últimos.

De este modo, el análisis del concepto americanismo que deriva de la aplicación de dos supuestos claves -a saber, su definición exclusiva a partir del uso (Company, 2007: 28-9) y su carácter dinámico (Ramírez Luengo, 2012: 398)- permite establecer las pautas históricas que determinan la creación de estos elementos y, como consecuencia, una taxonomía que sistematiza los diversos subtipos que es posible distinguir cuando se atiende a su diacronía; en concreto, la taxonomía propuesta a lo largo de estas páginas es la siguiente:

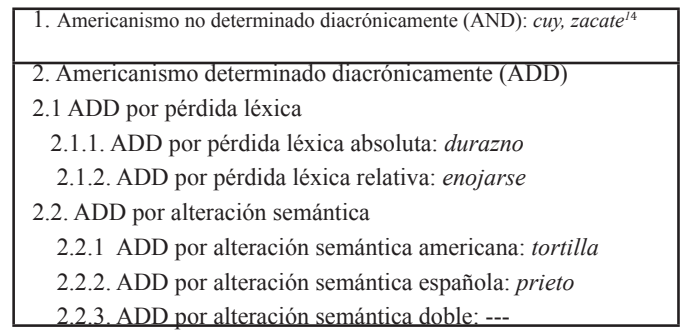

Tabla 2: Taxonomía de los americanismos según sus pautas diacrónicas de creación

Llegados a este punto, se hace necesario hacer hincapié una vez más en que el interés de una taxonomía como la presente va más allá de un mero afán clasificatorio que se agota en sí mismo; muy al contrario, lo que se pretende con ella es dotar al investigador de un primer marco explicativo que atienda a las particularidades que presentan los americanismos en su perspectiva diacrónica y que permita analizarlos atendiendo a sus propias pautas históricas de creación, en la idea de que tal marco explicativo ha de redundar sin duda en un conocimiento más preciso de estos elementos $\mathrm{y}$, por tanto, de la propia configuración del léxico diferencial que caracteriza actualmente al español de América.

\section{Conclusiones}

El carácter de primera aproximación a la materia y ensayo de método con que se ha querido dotar al presente trabajo hace inevitable que, si no todas, la mayor parte de las conclusiones que se desprenden de él no constituyan de hecho sino nuevos interrogantes y posibles líneas de trabajo que será necesario investigar en un futuro más o menos próximo.

De este modo, la labor más inmediata ahora es aplicar las pautas diacrónicas señaladas a lo largo de estas páginas al estudio de las diversas unidades léxicas que en estos momentos se consideran americanismos, con el propósito de determinar no solo a qué subtipo de la taxonomía propuesta pertenecen, sino también -y muy especialmente- su particular historia, es decir, los procesos que han conducido a la situación actual que cada uno de estos vocablos presenta y la cronología concreta en que han tenido lugar.

Al mismo tiempo -y como consecuencia inevitable de lo anterior-, se hace también necesario extraer una serie de principios generales que permitan trascender la mera historia de las palabras para llegar a una historia del léxico; en esta línea, el investigador tendrá que dar respuesta a cuestiones como, por ejemplo, qué pauta diacrónica de creación de americanismos resulta más habitual en la historia del español, qué factores -la etimología del vocablo, el momento en que ocurre el punto de modificación valorativa, otros distintos...- favorecen el empleo de una u otra pauta, o las características de todo tipo 
que, más allá de su propia configuración histórica, particulariza a cada uno de los esquemas de creación propuestos.

Por otro lado, constituye una cuestión no menor establecer la relación que existe entre las pautas aquí propuestas y los tres tipos de americanismos postulados por Company (2010: XVII): aunque es preciso reflexionar aún más sobre la cuestión y justificar con datos concretos las conclusiones a las que se llegue, parece en principio lógico pensar que, mientras que los AND necesariamente han de ser americanismos puros, los ADD engloban tanto elementos pertenecientes a esta categoría como muy especialmente aquellos que la profesora mexicana denomina americanismos semánticos y americanismos de frecuencia; a este respecto, además, se convierte en una tarea fundamental examinar desde esta perspectiva la última categoría mencionada, que de por sí presenta dificultades teóricas y prácticas de gran interés (Ramírez Luengo, en prensa) y cuyo análisis diacrónico quizá pueda resultar un poco más claro a la luz de los postulados que se defienden en estas páginas.

Por último -y probablemente más marginal respecto a los aspectos anteriores-, conviene no olvidar otro punto de importancia para la cabal comprensión de las transformaciones que afectan históricamente al léxico del español: del mismo modo que crean americanismos, el carácter dinámico de este concepto y los procesos de extensión léxica ya mencionados producen también el resultado contrario, esto es, la desamericanización de determinadas unidades léxicas $o$, si se quiere, su cambio de estatus de americanismos a voces generales (Ramírez Luengo, 2014: 3). Pues bien, también sería oportuno analizar con calma este asunto y sus propias peculiaridades -desarrollo preciso, voces afectadas, momentos cronológicos de cambio de estatus...- a la luz de lo expuesto anteriormente, habida cuenta de que tales procesos constituyen el reverso de los fenómenos que se están analizando en estas páginas $\mathrm{y}$, como tal, su mejor comprensión puede facilitar claves que ayuden a clarificar los numerosos interrogantes planteados hasta el momento.

Frente a todas las cuestiones que quedan abiertas por el momento para posteriores reflexiones, quizá el presente análisis tenga el mérito de ofrecer al menos dos certezas incuestionables: por un lado, que existen diferentes pautas diacrónicas que explican la creación de los diversos americanismos que se pueden descubrir en una sincronía dada; por otro, que es imprescindible acercarse a estos elementos con una metodología adecuada y rigurosa que parta de los propios rasgos definitorios de este concepto y tenga en cuenta todos los aspectos específicos que lo caracterizan en su dimensión diacrónica, pues solo de esta manera -más compleja, sin duda; pero también más realista- será posible obtener una visión esclarecedora y ajustada a la realidad histórica de sus procesos de creación y desarrollo; de los pasos, en definitiva, que, a través del tiempo y a lo largo de cinco siglos, hacen posible el establecimiento y la consolidación de la enorme riqueza y diversidad léxica que identifica hoy de manera inconfundible a las diversas variedades que conforman el español que se habla en América. 


\section{Referencia Bibliográfica}

Bohórquez, J. G. (1984). Concepto de americanismo en la historia del español. Bogotá: Instituto Caro y Cuervo.

Buesa Oliver, T. y Enguita Utrilla, J. M. (1992). Léxico del español de América: su elemento patrimonial e indígena. Madrid: Mapfre.

Company, C. (2007). El siglo XVIII y la identidad lingüística de México. Discurso de ingreso a la Academia Mexicana de la Lengua (10 de noviembre de 2005). México DF: Universidad Nacional Autónoma de México / Academia Mexicana de la Lengua.

Company, C. (2010). Introducción. En Academia Mexicana de la Lengua: Diccionario de Mexicanismos (XV-XXIII). México DF: Siglo XXI.

CORDE. Real Academia Española (2015). Banco de datos CORDE. Corpus Diacrónico del Español. Recuperado de: <http://corpus.rae. es/cordenet.html>.

CREA. Real Academia Española (2015). Banco de datos CREA. Corpus de Referencia del Español Actual. Recuperado de: <http:// www.rae.es>.

DAM. Asociación de Academias de la lengua española (2010). Diccionario de Americanismos. Madrid: Santillana.

Donadío Copello, M. (2005). Algo acerca de los americanismos y las regiones dialectales. En Aráus Puente, C. (coord.). Manual de lingüística hispanoamericana, II (83-141). Bogotá: Instituto Caro y Cuervo.

Frago, J. A. (1999). Historia del español de América. Madrid: Gredos.

Hildebrant, M. (1961). La lengua de Bolívar, I. Léxico. Caracas: Universidad Central de Venezuela.

Mejías, H. A. (1980). Préstamos de las lenguas indígenas en el español americano del siglo XVII. México DF: Universidad Nacional Autónoma de México.

Moreno de Alba, J. G. (1992). Diferencias léxicas entre España y América. Madrid: Mapfre.
Moreno de Alba, J. G. (2007). Introducción al español americano. Madrid: ArcoLibros.

Ramírez Luengo, J. L. (2012). El léxico en los procesos de dialectalización del español americano. Cuadernos del Instituto de Lengua Española, 7, 393-404.

Ramírez Luengo, J. L. (2014). Cómo el español de España genera americanismos: a propósito del americanismo puto 'homosexual'. Lengua y habla, 18, 1-12.

Ramírez Luengo, J. L. (en prensa). Aspectos metodológicos para el estudio histórico del léxico americano: conceptos, ejemplificación y tareas para el futuro. En Huisa Téllez, J. C. (ed.). El léxico del español americano y su estudio histórico. Hacia la revisión de las fuentes (en prensa). Berna: Peter Lang.

Vaquero de Ramírez, M. (1996). El español de América, II. Morfosintaxis y léxico. Madrid: ArcoLibros.

\section{Notas al lector}

1 Se define esta dialectalización como el 'resultado de los procesos de selección normativa que conllevan la imposición (o el rechazo) de determinados fenómenos que identifican y caracterizan geográficamente el español de una región' (Ramírez Luengo, en prensa); véase este trabajo para algunas precisiones a la definición inmediatamente facilitada.

2 Como se recuerda en Ramírez Luengo (en prensa), el estudio no puede reducirse al análisis de las unidades léxicas que configuran esos "mapas identificadores" ya mencionados, sino que se ha de ampliar también a cuestiones tan variadas como el momento en que la concreción de tales mapas tiene lugar y los procesos que los conforman, así como -muy especialmente- a los factores (socio)históricos que determinan tales configuraciones, en ocasiones notablemente diferenciadas entre sí.

3 Para una completa revisión historiográfica de las interpretaciones que se han dado a este concepto, véase el trabajo de Donadío Copello (2005: 89-92).

4 En concreto, americanismos puros ('voces empleadas en el español general de América 
inexistentes en el español peninsular general'), americanismos semánticos ('voces y construcciones formalmente compartidas con el español peninsular, pero que han desarrollado en América valores semánticos propios') y americanismos de frecuencia ('voces o construcciones compartidos, en forma y significado, con el español peninsular castellano, pero que muestran en América una mucho mayor frecuencia de empleo y de generalización') (Company, 2010: XVII).

5 Para un análisis detallado de las consecuencias que se derivan de los aspectos mencionados en un corpus histórico del siglo XVIII, véase Ramírez Luengo (en prensa).

6 Entiéndase extensión léxica en este caso como 'todo proceso (histórico) de modificación en la distribución geográfica de una voz', sea de expansión (generalización) o de reducción (dialectalización)' (Ramírez Luengo, 2014: 4).

7 En Ramírez Luengo (2014) se aplica la perspectiva inmediatamente descrita al análisis de un caso específico -la voz puto con su valor de 'homosexual'-, y se demuestra cómo este elemento, actualmente americanismo semántico, solo adquiere tal valoración en un momento histórico muy concreto (el siglo XVIII), precisamente cuando su empleo con tal significación desaparece de las variedades peninsulares del español.

8 Y tomando necesariamente como eje del análisis, por supuesto, la situación existente en la sincronía que se está considerando en el estudio, término cronológico de referencia que -en principio- el estudioso ha de emplear en todas sus investigaciones.

9 No hace falta decir que -por una serie de cuestiones que se plantearon ya en Ramírez Luengo (en prensa)- el empleo de CORDE no resulta del todo satisfactorio a la hora de llevar a cabo estudios como el que aquí se pretende; sin embargo, no cabe duda de que la riqueza de sus fondos lo convierte por el momento en el instrumento más adecuado con el que puede contar el investigador interesado en estas cuestiones.

10 Desde el punto de vista sincrónico, en CREA (Consulta: 09/04/2015) no se registra ni una sola atestiguación española de ambos términos; en el caso de la diacronía, CORDE
(Consulta: 09/04/2015) aporta unos pocos ejemplos -22 de cuy y 15 de zacate, datados en su totalidad entre los siglos XVI y XIXque clasifica como españoles, pero lo cierto es que en todas las ocasiones aparecen en obras referidas al Nuevo Mundo -al estilo de la Historia del Nuevo Mundo, de B. Cobo, o la Historia natural y moral de las Indias, de J. de Acosta-, de manera que su aparición parece estar determinada por la tipología y muy especialmente la temática de las fuentes, y no tanto por ser palabras de empleo conocido en el español europeo; esto justifica la consideración de ambos vocablos como americanismos no determinados diacrónicamente (AND).

11 Hay que indicar que en este caso dos de los ejemplos que CORDE considera españoles aparecen en los Estudios Lingüísticos de Amado Alonso, en concreto en los trabajos sobre del español de América que se agrupan en sus Temas Hispanoamericanos; parece necesario, por tanto, extraer tales ejemplos de los porcentajes facilitados, lo que modifica levemente los datos anteriores; con esta nueva perspectiva, los usos españoles se reducen a un $12,82 \%$ y los americanos ascienden hasta el $87^{\prime} 17 \%$ del total.

12 En línea con lo mencionado en la nota 10, y tal y como se indicaba en Ramírez Luengo (en prensa), el hecho de que CORDE constituya un corpus cuantitativamente dispar desde el punto de vista diatópico -con una presencia muy reducida de textos americanos en comparación con los españoles- en parte resta credibilidad a los porcentajes precisos que se extraen de él, de manera que, como principio general de análisis e interpretación de sus datos, se puede sostener que "la aparición de un mayor número de ejemplos de determinado elemento léxico en América (...) puede ser un claro indicio de su empleo más frecuente en este continente" (en este caso, siglos XVIII, XIX y XX), pero "un número más elevado de ejemplos en España no resulta en modo alguno significativo, pues puede ser simple consecuencia del mayor volumen de texto español en el corpus y no tanto reflejo de la realidad de uso en la época" (en el ejemplo, siglos XVI y XVII). De ahí que en este caso interesen no tanto los datos concretos que muestra la tabla, sino el clarísimo cambio de tendencia en el uso que, de forma coherente y continuada, parece descubrirse en ella. 
13 Se entiende por pérdida absoluta aquella que produce la desaparición de un determinado elemento léxico en España o que, en última instancia, reduce su empleo a meras apariciones anecdóticas o regionales; por el contrario, la pérdida relativa no conlleva resultados tan radicales, sino simplemente su reducción de empleo en esta variedad respecto a lo que constituye la normalidad americana y su transformación, por tanto, en un americanismo de frecuencia; categoría, por cierto, no carente de problemas teóricos de notable importancia en cuanto a su determinación que aún es necesario analizar detalladamente.

14 Aunque esta categoría se ejemplifique con indigenismos, téngase en cuenta que los elementos léxicos que se engloban en ella no han de ser necesariamente de este origen, habida cuenta de la existencia de voces formadas por derivación a partir de bases patrimoniales que también cumplen con las características ya mencionadas para incorporarse a ella. 